\title{
Regeneration of Vascular Wall Based on the Suture
}

Abildayev DA, Semzhanova ZA, Singh T, Kumar LDP*, Singh C and Naveen C

Department of Clinical Anatomy and Operative Surgery, Kazakh-National Medical University, Kazakhstan

*Corresponding author: Kumar LDP, Department of Clinical Anatomy and Operative Surgery, International Medical Faculty, Kazakh-National Medical University, Kazakhstan, Tel: 77273387090; E-mail: pawankarthik09@gmail.com

Received date: December 4, 2018; Accepted date: January 3, 2019; Published date: January 10, 2019

Copyright: (c) 2019 Abildayev DA, et al. This is an open-access article distributed under the terms of the Creative Commons Attribution License, which permits unrestricted use, distribution and reproduction in any medium, provided the original author and source are credited.

\begin{abstract}
Authors performed an updated and thorough review of the literature regarding regeneration of vascular wall based on the various suture and their healing properties. A comprehensive literature review was conducted and written by using the keywords anastomosis, regeneration, biodegradable polymers. We described data on the different sutures and how healing occurs. It was possible to synthesize the current knowledge about present sutures and present their peculiarities according to examined laboratory findings.
\end{abstract}

Keywords Anastomosis; Regeneration; Vascular surgery

\section{Introduction}

Vascular surgery deals with blood vessels, these vessels can vary in size and length. Bleeding associated with surgery is a potentially serious complication and it's difficult for regeneration and healing so hemostasis to prevent blood loss is commonly achieved by tying a surgical thread around the blood vessel by applying different vascular sutures at the zone of injury [1]. The principles of vascular repair with sutures were established in the first decade of the $20^{\text {th }}$ century by Alexis Carrel, who in 1912 was awarded the Nobel Prize for Medicine for this work. Since from then, many refinements of suture materials and types of sutures have made possible surgical reconstruction of most arteries, from the root of the aorta to microvascular anastomosis or repair of the smallest vessels, e.g. digital arteries or those on the surface of the brain. Fine sutures on atraumatic needles are best for arterial anastomosis. Silk was used for many years, but it has now been replaced by synthetic fibers, which are less traumatic to the vessel walls [2].

Knowledge of Carrel's work spread rapidly and practical application of his work was reflected in the development of vascular replacements, such as venous grafting, the bypass technique used for vein grafts before World War I. There was, however, a lag in further developments until the end of the forties when dos Santos and Kunlin revived old methods and opened the way for further arterial surgical advances [3].

\section{Material and Methods}

By considering and analyzing various articles the method which we used is broadly a systematic analysis and review of the closely related articles from the libraries like clarivate, web of science, science direct, PubMed, Google Scholar, Cochrane Library using the keywords regeneration, sutures, Totally we found 22 related articles by From there we have chosen articles, which were relevant for our theme.

\section{Discussion and Result}

The true evolution of the use of the suture goes back to 1761 when Mr. Richard Lambert of Newcastle-upon-Tyne wrote to Dr. William
Hunter and described how Mr. Hallowell, at his suggestion, had opened a brachial artery aneurysm and found only a small tear in the artery as the source of an aneurysm [4]. At Mr. Lambert's suggestion, a small steel pin, " $/ 4$ " in length, was placed through the lips of the wound and a ligature wound about the pin approximating the wound and controlling bleeding instead of ligating the artery (Figure 1). This presumably allowed persistence of flow through the vessel because the patient suffered no significant diminution of the radial pulsation.

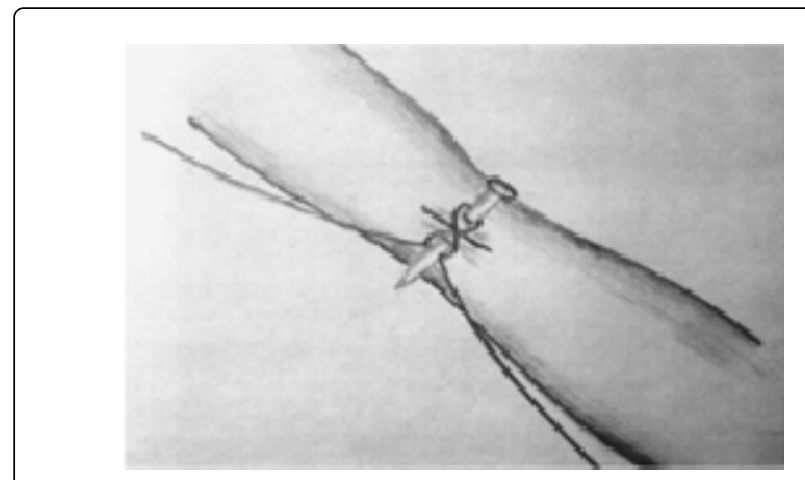

Figure 1: Sketch of pin closure of Lambert and Hallowell for small defect in brachial artery. This is modification of farrier's suture used in horses, as well as for approximation of hare lip.

When successful, both anastomotic techniques resulted in a wellvascularized transplant of the entire small intestine. Technically, it was found to be much easier to perform an anastomosis between the graft aorta and the recipient aorta than between the graft superior mesenteric artery and the recipient aorta because of the size of the vessels. Attempts to use a continuous suture for the latter anastomoses were unsuccessful mainly because of a tendency for the anastomosis to become stenosed when the suture was tied. It seemed that the increased bleeding problems encountered occurred principally from bleeding between the sutures [5].

Landon described a short metal cannula made in 3 sizes. One end of the cannula was smooth and the other end housed 5 sharp hooks that were slightly everted from the base. Either the vein or the artery was 
Citation: Abildayev DA, Semzhanova ZA, Singh T, Kumar LDP, Singh C, et al. (2019) Regeneration of Vascular Wall Based on the Suture.

Page 2 of 2

threaded through the cannula, and the end of the vessel was cuffed back and fastened to the hooks in a circular manner. The other end of the vessel was then pulled over the first end and placed on these hooks. "McGrath" devised transfusion forceps that could be separated and locked. At the end of each half, a short cannula similar to that of Landon 7 was attached. The blood vessel ends were threaded through the cannulae, cuffed back, and fastened to the hooks. The forceps were then brought together and gently locked, and the blood flow was turned on [6].

"Donetskii" devised a stainless steel ring resembling that of Landon in that it had several spikes which could be utilized to hook on the edges of the vessels, thus enabling the surgeon to pursue end-to-end, end-to-side, and side-to-side anastomosis of small blood vessels (Figure 2) [7].

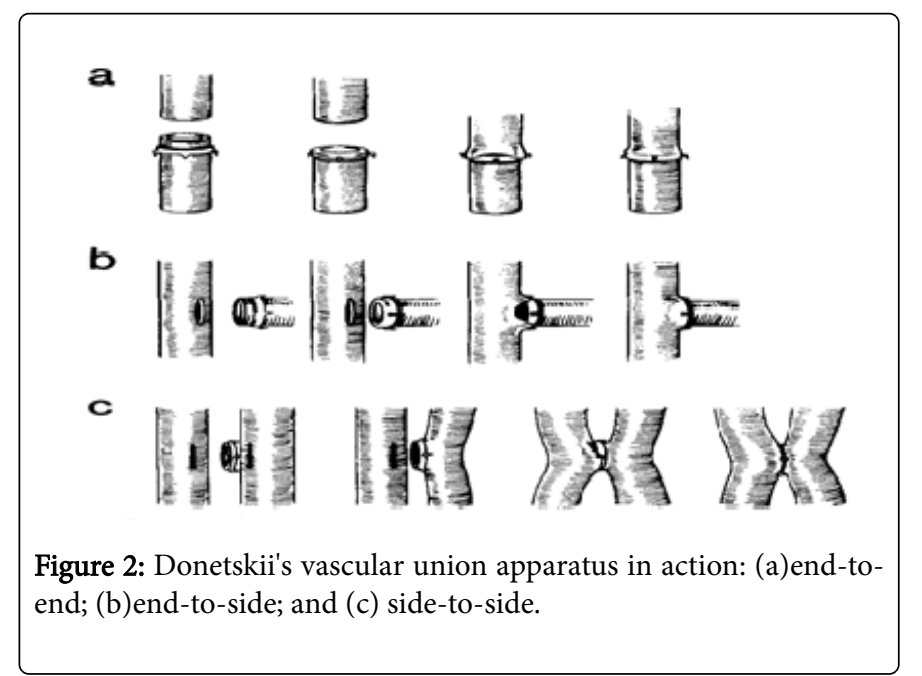

\section{Conclusion}

Various non-suture anastomotic techniques are currently available. At present, the benefits of stents and glues hardly outweigh their disadvantages. Lasers need further development before routine clinical use. Worldwide, the two systems that have been used most frequently as alternatives to sutures in the past decade are the unilink system and the VCS clip applier system. Both are available and both secure an easy and rapid anastomosis. Furthermore, they can be used for arteries and for veins in either an end-to-end or an end-to-side configuration. So we conclude that non sutures techniques are easily regenerative than normal.

\section{References}

1. Kudur MH, Pai SB, Sripathi H, Prabhu S (2009) Sutures and suturing techniques in skin closure. Indian J Dermatol Venereol Leprol 75: 425-434.

2. PL Harris (1994) Arterial suture and anastomosis. Vascular Surgery, Springer, Boston, MA.

3. Wiley F Barker (1988) A century's worth of arterial sutures. Ann Vasc Surg 2: 85-91.

4. Erichsen JE (2007) Observations on aneurism: Selected from the works of the principal writers on that disease, from the earliest periods to the close of the last century. London Sydenham Society, 1844.

5. Muste A, Beteg F, Ober CA, Briciu R, Donisa A, et al. (2008) The vascular sutures and anastomosis in dog. Sci Vet Med work 41: 220-226.

6. FA Weaver, BD Hood, AE Yellin (2000) Vascular injuries of the extremities, in Rutheford RB "Vascular Surgery". Philadelphia, WB Saunders, pp: 862-870.

7. SP John Lumley (1986) Colour atlas of vascular surgery. Wolfe Medical Publications, London. 